\title{
Time-Resolved X-Ray Absorption Spectroscopy of Infrared-Laser Induced Temperature Jumps in Liquid Water
}

\author{
G. Gavrila ${ }^{1}$, K. Godehusen ${ }^{1}$, C. Weniger ${ }^{1}$, E. T. J. Nibbering ${ }^{2}$, \\ T. Elsaesser ${ }^{2}$, W. Eberhardt ${ }^{1}$, Ph. Wernet ${ }^{1}$ \\ ${ }^{1}$ Helmholtz-Zentrum Berlin für Materialien und Energie GmbH, Albert-Einstein-Str. 15, D-12489 Berlin, Germany \\ ${ }^{2}$ Max-Born-Institut für Nichtlineare Optik und Kurzzeitspektroskopie,Max-Born-Str. 2 A, D-12489 Berlin, Germany \\ Corresponding author email: wernet@bessy.de
}

\begin{abstract}
A time-resolved x-ray absorption study of the structural dynamics of liquid water on a picosecond time scale is presented. We apply femtosecond mid-infrared pulses to resonantly excite the intramolecular $\mathrm{O}-\mathrm{H}$ stretching band of liquid water and monitor the transient response in the oxygen K-edge absorption spectrum with picosecond $\mathrm{x}$-ray pulses. In this way, structural changes in the hydrogen bond network of liquid water upon an ultrafast temperature jump of approximately 20 Kelvin are investigated. The changes of the x-ray absorption as induced by such a temperature jump are about $3.2 \%$. This demonstrates that our method serves as a sensitive probe of transient structural changes in liquid water and that combined infrared laser-synchrotron experiments with substantially shorter $\mathrm{x}$-ray pulses such as generated with a femtosecond slicing scheme are possible.
\end{abstract}

\section{Introduction}

For structure determination of matter, $\mathrm{x}$-ray techniques have been the most commonly used and the most accurate. Until recently they have primarily been performed under stationary or quasi-stationary conditions addressing time-averaged equilibrium structures. An extension of x-ray methods into the ultrafast time domain is highly desirable since it enables unprecedented insight into transiently evolving matter by allowing direct probing of rapidly changing structures. The dynamic properties of liquids and solids are coupled to atomic motions and the relevant time-scale is that of a vibrational period $[\leq 100$ femtoseconds $(\mathrm{fs})=$ $\left.10^{-13} \mathrm{~s}\right]$. This is the time scale on which molecular dynamics, the making and breaking of chemical bonds, the rearrangements of atoms and phase transitions occur. One case where the understanding of real-time nuclear dynamics has attracted much attention is that of liquid water.

Ordinary yet unique, liquid water is probably the most important substance on earth. It possesses a number of peculiar properties that are assumed to be essential for life in any form known today, and its complex molecular structure determines these properties [1]. Liquid water consists of an extended molecular network with highly polar water molecules that are coupled via intermolecular hydrogen bonds. The time-averaged equilibrium structure is most commonly probed with neutron and $\mathrm{x}$-ray diffraction in terms of radial distribution functions 
$[2,3]$ and with infrared spectroscopy [4-8]. Recently, x-ray spectroscopy has been applied to liquid water [9-24] and in particular x-ray absorption spectroscopy (XAS) studies have initiated a debate about the local structure of liquid water [9,19-28]. These investigations also addressed time-averaged equilibrium structures such as average local configurations of hydrogen bonds. However, the hydrogen-bonded network in liquid water is continuously changing and a more profound understanding of liquid water may be obtained by following its hydrogen bonded dynamical structure in real time.

The structural dynamics of liquid water including the breaking and making of hydrogen bonds occur on femto- to picosecond time scales set by the strength of intermolecular interactions and the different vibrational and translational motions [29-30]. Ultrafast vibrational spectroscopies provided detailed information on the coupling between inter- and intramolecular vibrations and the resulting ultrafast processes [4-8]. However, these transient vibrational spectra only give indirect insight into time-dependent water structure. A combination of ultrafast vibrational and $\mathrm{x}$-ray spectroscopies therefore promises to give unprecedented insight into the structure of liquid water.

Ultrafast time-resolved $\mathrm{x}$-ray science [31-50] is an emerging field that is now undergoing rapid expansion owing to technological advances in the generation of ultrashort xray pulses. Ultrafast $\mathrm{x}$-ray science is currently performed with short-pulse $\mathrm{x}$-ray sources falling into two main categories: accelerator-based [31-40] and laser-driven table top sources [41-49]. In combination with femtosecond lasers these have been applied for time-resolved xray diffraction, scattering and spectroscopy. For the study of molecular dynamics picosecond XAS has successfully been used in particular to probe transient structures of electronically excited molecules $[31,33,36,40]$.

Here we present our approach to unravelling structural dynamics in liquid water by applying a time-resolved x-ray probe technique in combination with an ultrafast infrared pump. We use femtosecond (fs) infrared excitation via the O-H stretching band to transfer energy into the vibrational system, which after the relaxation and redistribution can effectively be described as a temperature jump on a time scale of a few picoseconds. The concomitant changes in the hydrogen bond network are probed with transient XAS at the oxygen $(\mathrm{O}) \mathrm{K}$ edge with $\mathrm{x}$-ray pulses from a storage ring source to probe these changes. In a first publication we reported a picosecond mid-infrared pump - x-ray probe study with relative changes of the $\mathrm{x}$-ray absorption of less than $0.2 \%$ [51]. This demonstrated the high sensitivity to detect the weakening or breaking of hydrogen bonds as due to the ultrafast temperature jump with time-resolved XAS and the stability of our pump-probe experiment. It 
also represented the first mid-infrared pump - x-ray probe study of ultrafast structural fluctuations in the electronic ground state of a molecular liquid.

In this paper, we report on our recent progress using this approach, where we now probe relative changes of the $\mathrm{x}$-ray absorption as large as $\sim 3.2 \%$ hence demonstrating the feasibility of such combined mid-infrared laser-synchrotron experiments using substantially shorter x-ray pulses such as generated with a femtosecond slicing scheme.

\section{Experiment}

Applying XAS to liquid water has been a long-standing challenge due to the incompatibility of the high vapour pressure of the liquid and the high-vacuum conditions required for XAS in the soft X-ray energy range. Typical approaches include measurements of the total fluorescence yield at a pressure of 1 bar of $\mathrm{He}$ [18], the electron yield on a liquid jet in vacuum [52] and x-ray absorption in transmission mode on thin films in x-ray transparent cells [53]. We measure the $\mathrm{O} \mathrm{K}$-edge absorption of water in transmission mode with liquid water confined in a cell (Fig. 1). Using the transmission mode proved to be the most reliable way of measuring this spectrum with soft x-rays since it was shown to agree with the reference spectrum from hard x-ray Raman scattering [53, 54]. However, due to the short penetration depth of soft x-rays in water, the sample thickness needs to be limited to a few hundred nanometers to allow sufficiently high output signals for appropriate data interpretation. This also ensures maximum efficiency for mid-infrared pump and soft x-ray probe spectroscopy of water since virtually the whole excited volume in the sample is also probed with $\mathrm{x}$-ray absorption spectroscopy at the $\mathrm{O}$ K-edge. The absorption lengths (reduction of the incident intensity by $1 / \mathrm{e}$ ) in water of both mid-infrared radiation in the center of the $\mathrm{O}$ stretch vibrational band $\left(3400 \mathrm{~cm}^{-1}\right)$ and of soft x-rays at the $\mathrm{O} \mathrm{K}$-edge $(550 \mathrm{eV})$ match very well $(800 \mathrm{~nm}$ in the $\mathrm{O}-\mathrm{H}$ stretch band and $600 \mathrm{~nm}$ at the $\mathrm{O} \mathrm{K}$-edge).

The cells used to prepare the nanometer thin liquid water films are based on $\mathrm{x}$-ray transparent $\mathrm{Si}_{3} \mathrm{~N}_{4}$ membranes supported by $\mathrm{Si}$ wafers. Membranes of various thicknesses (100 to $300 \mathrm{~nm}$ ) and sizes were developed and tested at the Application Center for Microengineering at BESSY. Metallic spacers are used to keep the membranes at a defined distance and to control the water film thickness (Fig. 1 (b)). The data shown here were taken with $\mathrm{Si}_{3} \mathrm{~N}_{4}$ membranes with a thickness of $300 \mathrm{~nm}$ and a size of $800 \times 800 \mu \mathrm{m}^{2}$. The metallic spacers had a height of $250 \mathrm{~nm}$. Cells were filled by sandwiching a droplet of deionized water between two membranes. After carefully squeezing the excess water out of the cell through the channels in the spacer (Fig. 1 (b)) the edges of the Si wafers were sealed using either 
proper adhesive technology or by using viton O-ring gaskets together in a mechanical mount. The water film thickness in our cell for the transient x-ray absorption measurements (Fig. 5) was determined to approximately $600 \mathrm{~nm}$ by comparison of the measured to the calculated $\mathrm{x}$ ray transmission as demonstrated in Fig. 2 (a).

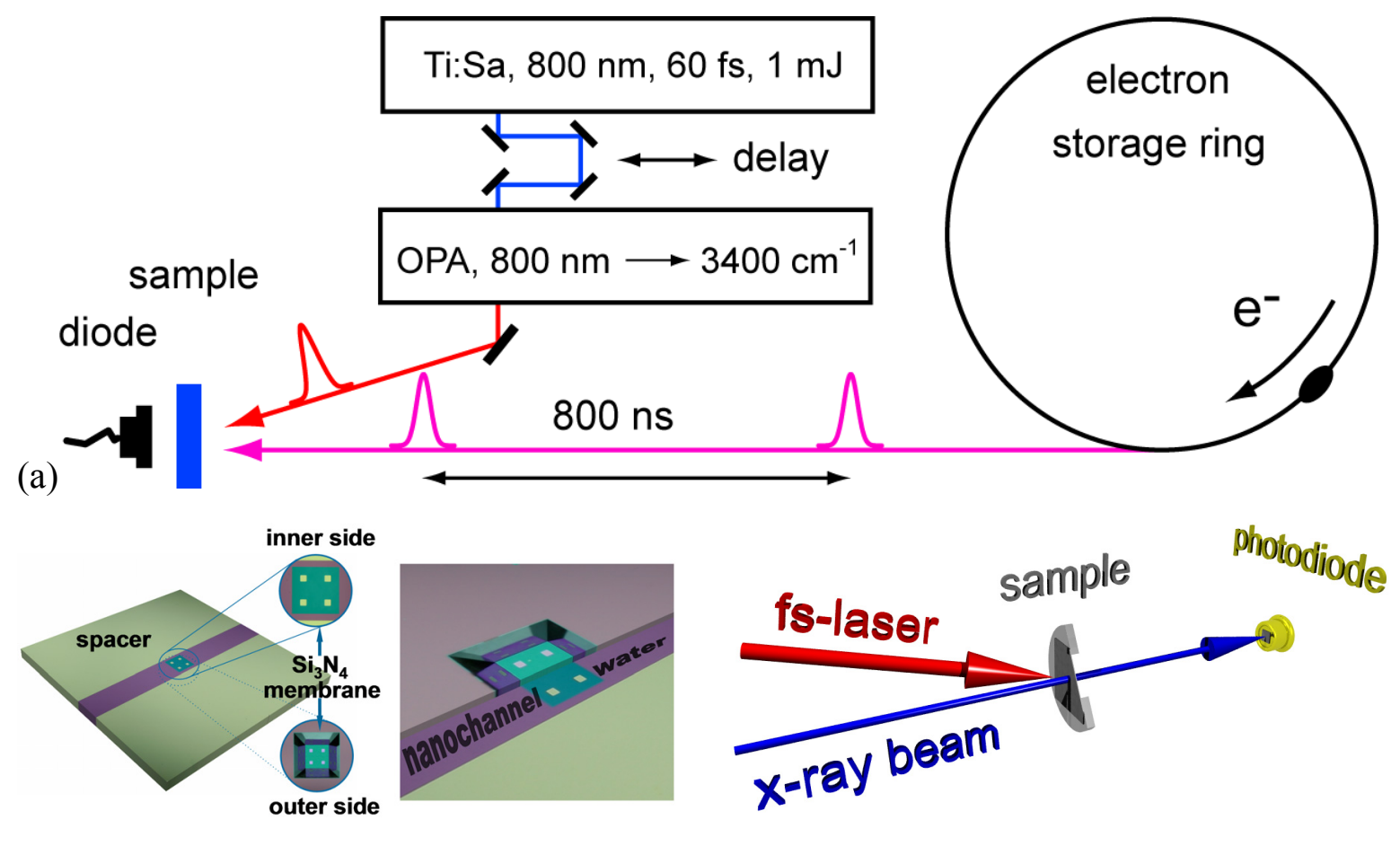

(b)

(c)

Fig. 1. (a) Experimental set-up (b) The nanostructured $\mathrm{Si}_{3} \mathrm{~N}_{4}$ membranes on the Si support wafer with metallic spacers used for building the liquid water sample cell. (c) Close-up of the interaction region.

Amorphous ice was prepared by condensing in ultrahigh vacuum deionized water onto a $\mathrm{Si}_{3} \mathrm{~N}_{4}$ membrane that was cooled to liquid Nitrogen temperature. The crystalline ice sample was made by slowly heating the amorphous sample to about $150 \mathrm{~K}[14,55]$. Crystallization was observed to be complete after approximately 30 minutes. The ice samples were $200 \mathrm{~nm}$ thick.

Steady-state x-ray absorption spectra were taken by detecting the transmitted x-ray flux with a GaAs diode both in hybrid- and single-bunch mode of the electron storage ring BESSYII (Fig. 1(c)). The hybrid-bunch mode at BESSYII consists of 350 consecutive electron bunches spaced by $2 \mathrm{~ns}$ and a single bunch placed in a gap of $100 \mathrm{~ns}$. For transient $\mathrm{x}-$ ray absorption x-ray pulses were detected with a fast avalanche photodiode (APD) (Fig. 1(c)). Time-resolved detection with box car integrators allowed us to record the transmitted x-ray 
signal both in single and hybrid bunch mode with and without infrared excitation with consecutive x-ray pulses from the single bunches $800 \mathrm{~ns}$ apart from each other and, hence, each at a repetition rate of $1 \mathrm{kHz}$ (Fig. 1 (a)). The duration of the x-ray pulses was typically 80 ps. Transient x-ray absorption was measured by detecting the transmitted x-ray flux at fixed photon energy with variable pump-probe delays or at a fixed delay and by varying the $\mathrm{x}$ ray photon energy across the $\mathrm{O}$ K-edge. All spectra shown here are taken with a photon energy bandwidth of between 0.1 and $0.2 \mathrm{eV}$. The x-ray pulses were generated in the soft $\mathrm{x}-$ ray undulator beamline UE56-b at BESSYII.

The infrared pulses were generated in an optical parametric amplifier using an amplified femtosecond Ti:sapphire laser system $(1 \mathrm{kHz}$ repetition rate, $0.8 \mathrm{~mJ} /$ pulse, $60 \mathrm{fs}$ pulse duration). The mid-infrared radiation was tuned to the center of the $\mathrm{O}-\mathrm{H}$ stretching band at $3400 \mathrm{~cm}^{-1}$. Taking into account losses due to reflection on the vacuum window and on the $\mathrm{Si}_{3} \mathrm{~N}_{4}$ membrane the infrared pulse energy was measured to be $2.25 \mu \mathrm{J}$ at the water film. The infrared pulse duration was estimated to $120 \mathrm{fs}$. The pump beam entered the vacuum chamber at an angle of $30^{\circ}$ with respect to the x-ray beam propagation axis and was focused with a $\mathrm{f}=17 \mathrm{~cm}$ lens onto the sample.

The measured sizes of the pump and probe beams are shown in Fig. 2 (b) and (c). A quadratic pinhole $\left(300 \times 300 \mu \mathrm{m}^{2}\right)$ was scanned at the sample location through both the infrared and the x-ray beams and the transmitted intensity was measured with a powermeter and a GaAs photodiode, respectively. The data had to be slit deconvoluted because the pinhole size was similar to the spot sizes. We assume a round pump beam spot and Gaussian intensity distributions for both the pump and the probe beams. The x-ray beam spot is rectangular. According to the fit in Fig. 2 (b) the pump beam has a full width at half maximum (FWHM) of $132 \mu \mathrm{m}$. For the commonly used definition of beam width (the width at which the beam intensity has decreased to $1 / e^{2}$ or $13.5 \%$ of its peak value) the diameter is $210 \mu \mathrm{m}$. 

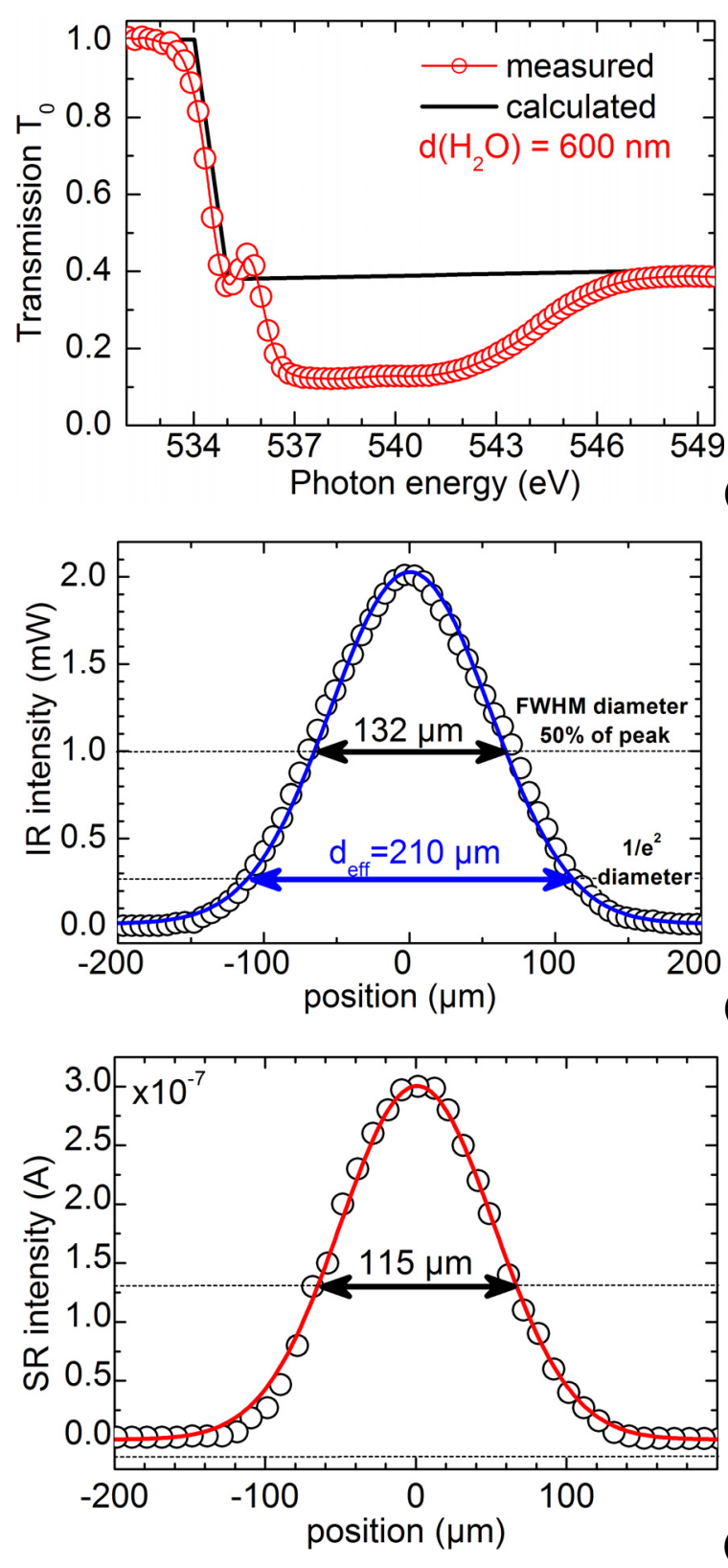

(b)

(c)
Fig. 2. (a) X-ray transmission spectrum of liquid water with calculated transmission for a water film thickness of $600 \mathrm{~nm}$ [56]. (b) Infrared (pulse power) and (c) Horizontal $\mathrm{x}$-ray beam spot sizes (diode current). Data from profiling measurements are shown as markers and Gaussian fits are shown as solid lines.

\section{Results and Discussion}

O K-edge absorption spectra of crystalline ice, liquid water and gas-phase water are displayed in Fig. 3. We focus here on the near-edge region of the x-ray absorption spectrum (near edge x-ray absorption fine structure, NEXAFS [55]). While the extended high-energy region of the spectrum (extended x-ray absorption fine structure, EXAFS) can be used to extract O-O radial distribution functions $[58,59]$, the near-edge region reflects how the local electronic structure of the absorbing molecules is affected by hydrogen bonding. It hence gives indirect access to the local structure of water. The $\mathrm{O}$ K-edge absorption spectrum arises from transitions of $\mathrm{O} 1 \mathrm{~s}$ electrons into empty molecular orbitals of the probed water molecules. Transitions to these empty states are particularly sensitive to hydrogen bonding 
with the nearest neighbors and the spectrum reflects the superposition of spectral contributions from water molecules in various configurations $[11,14,15,60]$. This is demonstrated with the $\mathrm{O}$ K-edge spectra of ice, liquid and gas-phase water in Fig. 3. Hydrogen bonding can obviously be probed effectively with the $\mathrm{O}$ K-edge absorption spectrum. Note that our ice spectrum agrees well with formerly reported crystalline ice spectra $[16,61-63]$.

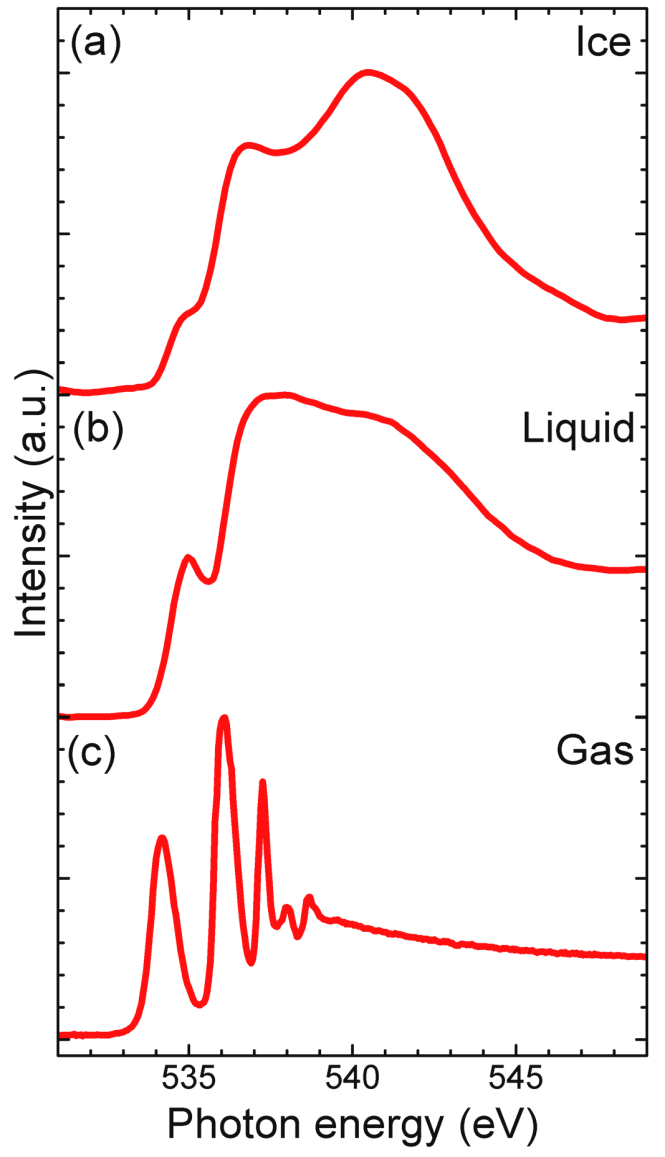

Fig. 3. Oxygen K-edge absorption spectra of (a) crystalline ice, (b) liquid water and (c) gas-phase water. The ice and liquid water spectra were measured in transmission mode (for details on the sample preparation see text). The gas-phase spectrum (total fluorescence mode) is taken from [18].

It is apparent that increasing the structural disorder, or the weakening and/or breaking of hydrogen bonds, in the condensed phases of water is indicated by increasing absorption in the so called pre- and main-edge regions of the spectra at $535 \mathrm{eV}$ and $537-538 \mathrm{eV}$, respectively, and decreasing absorption in the post-edge region (541-542 eV). The pre-edge and main-edge features are related to states localized around the species that are highly asymmetric with only one well defined hydrogen bond on the donor side. The post-edge feature, on the other hand, originates from more symmetric configurations with two strong donor hydrogen bonds. These three spectral features have been used before to characterize different hydrogen bonding configurations in water [9, 11, 14, 62]. 
A more detailed analysis of the liquid water x-ray absorption spectrum showed that varying the contribution of two structurally different configurations can explain these spectral changes $[9,11,54]$ : The increase of the relative amount of locally asymmetric configurations and the concomitant decrease of the relative amount of locally symmetric configurations is consistent with increasing absorption in the pre and main edges and with a decreasing absorption in the post edge. This model was also used to explain the changes in the $\mathrm{O} \mathrm{K}$-edge x-ray absorption spectra upon steady-state heating and cooling of liquid water [27] and the differences between liquid $\mathrm{H}_{2} \mathrm{O}$ and $\mathrm{D}_{2} \mathrm{O}$ [54]. It has also been used to interpret the steadystate temperature changes in the x-ray emission spectrum of liquid water [23]. In the $\mathrm{H}_{2} \mathrm{O}$ $\mathrm{D}_{2} \mathrm{O}$ comparison a quantum effect was found in addition to a change of populations of structurally different configurations as it occurs when changing the temperature. It was suggested that for a certain fraction of the molecules the asymmetry of their bonding configuration increases: One of the donor hydrogen bonds weakens while the other donor bond on the same molecule gets stronger. This increasing asymmetry in the hydrogen bonded network was recently confirmed in a neutron diffraction study [64].

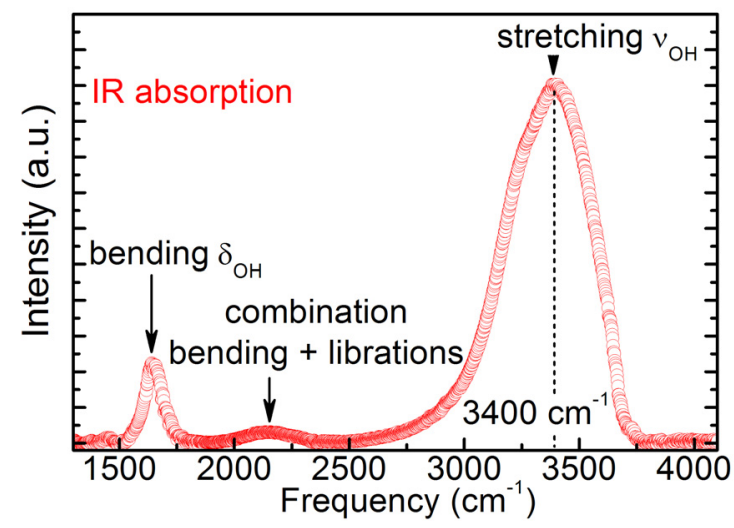

(a)

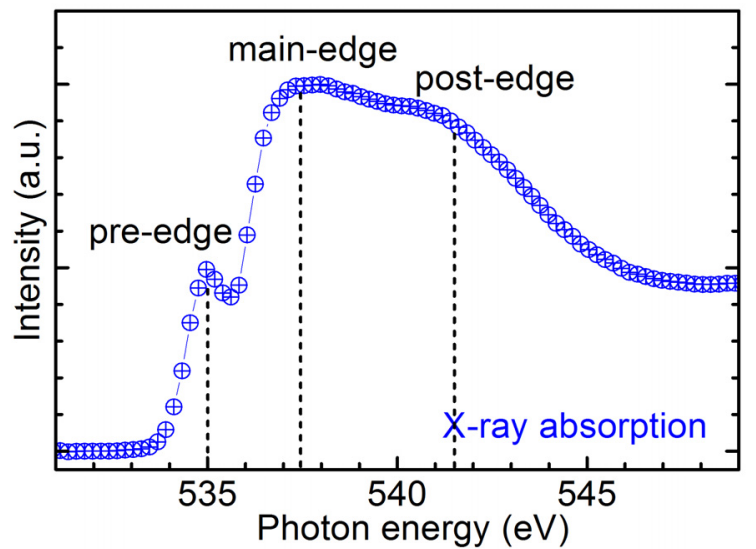

(b)

Fig. 4. (a) The infrared vibrational absorption spectrum of liquid water (b) x-ray absorption spectrum at the $\mathrm{O} \mathrm{K}$ edge of liquid water. Both spectra were taken on thin water films confined between two $\mathrm{Si}_{3} \mathrm{~N}_{4}$ membranes. Data are taken from [49].

The infrared and x-ray absorption spectra of liquid water are displayed in Fig. 4. The infrared spectrum was recorded in a commercial FTIR spectrometer. In all our time-resolved experiments the pump laser wavelength was tuned to the $\mathrm{O}-\mathrm{H}$ stretching band centered at $3400 \mathrm{~cm}^{-1}$. 
In Fig. 5 the steady state $\mathrm{O} \mathrm{K}$-edge transmission, $\mathrm{T}_{0}$, and the relative change of $\mathrm{x}$-ray transmission $\Delta \mathrm{T} / \mathrm{T}_{0}$ with $\Delta \mathrm{T}=\left(\mathrm{T}-\mathrm{T}_{0}\right)$ at two different photon energies near the pre-edge photon energy of $535 \mathrm{eV}$ are shown as a function of pump-probe delay time. The sample transmission decreases in a step-like fashion by approximately $3.2 \%$ at $534.6 \mathrm{eV}$ and $2.8 \%$ at $534.8 \mathrm{eV}$ and remains constant up to the longest delay time of 280 ps. The time evolution of the transmission decrease follows the time-integrated cross-correlation function of the femtosecond pump and the $\sim 80$ ps x-ray probe pulse (solid line), i.e., the width of the measured transmission step is determined by the duration of the probe pulses. We do not observe any long-lived transmission changes, suggesting a negligible accumulation of excess energy over a period of $1 \mathrm{~ms}$, the time interval between successive laser pulses.

In water, a femtosecond excitation around $2.94 \mu \mathrm{m}\left(3400 \mathrm{~cm}^{-1}\right)$ populates the $v=1$ state of the O-H stretching mode which displays a population decay with a time constant of $200 \mathrm{fs}$ [6-8]. The O-H stretching population decays via the $\mathrm{O}-\mathrm{H}$ bending mode and the excess energy is transferred into low-frequency intermolecular vibrations of the hydrogen bond network [7]. These ultrafast infrared spectroscopic studies demonstrate that the sample is fully equilibrated and macroscopically heated on a time scale of a few picoseconds with a larger fraction of intermolecular hydrogen bonds broken. We adopt the notion of temperature here even though we have not absolutely established that the population of vibrational and translational degrees of freedom below $400 \mathrm{~cm}^{-1}$ can be described by a thermal distribution.

The pre-edge transmission decrease observed around $535 \mathrm{eV}$ is the result of such transient heating and clearly indicates that the number of molecules in locally asymmetric configurations with one weak/broken hydrogen bond increases. As the duration of the x-ray probe pulses of $80 \mathrm{ps}$ is substantially longer than the intrinsic build-up time of the temperature jump, the time-resolved change of x-ray absorption follows the time integral of the cross correlation of pump and probe pulses.

The associated structural changes in liquid water upon mid-infrared excitation are characterized in more detail by means of transient XAS over the whole O K-edge at a fixed delay of 280 ps as shown in Fig. 6 (a). The transmission at the pre and main edges decreases while it increases at the post edge. We find in addition that the maximum relative change of $\mathrm{x}$ ray transmission is at a photon energy of $\sim 0.6 \mathrm{eV}$ below the water pre-edge absorption maximum. This corroborates the pump-probe measurements shown in Fig. 5 where the relative change of $\mathrm{x}$-ray transmission decreases when approaching the pre-edge maximum absorption energy. 

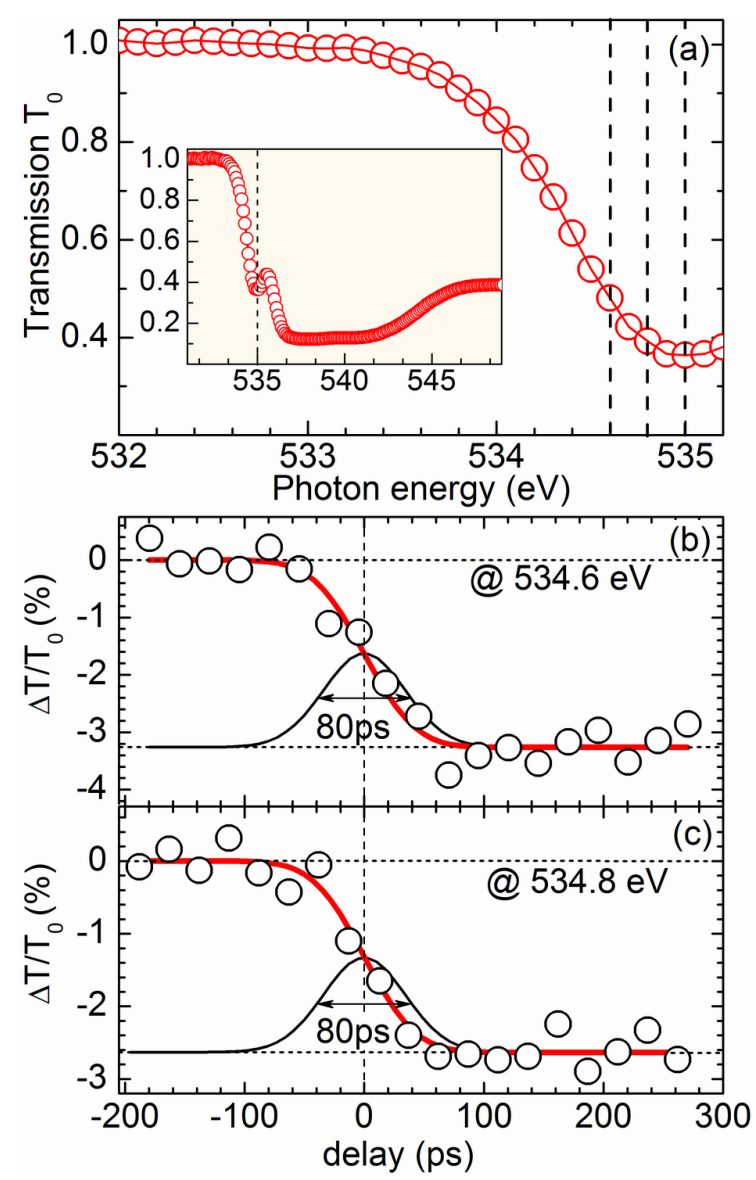

Fig. 5. (a) Steady-state transmission near the pre-edge photon energy $(535 \mathrm{eV})$. Inset: $\mathrm{O} \mathrm{K}$ edge x-ray transmission spectrum. (b) and (c) Change of x-ray transmission $\Delta \mathrm{T} / \mathrm{T}_{0}$ with $\Delta \mathrm{T}=\left(\mathrm{T}-\mathrm{T}_{0}\right)$ as a function of pump-probe delay time at the photon energies of $534.6 \mathrm{eV}$ and $534.8 \mathrm{eV}$, respectively. Measured data are shown as markers and the fit using a Gaussian (FWHM $=80$ ps) broadened step function is shown as solid line.

In order to understand these effects in more detail we compare the transient spectral difference in Fig. 6 (a) with a steady-state temperature difference (Fig. 6 (b)) and with the difference between $\mathrm{H}_{2} \mathrm{O}$ and $\mathrm{D}_{2} \mathrm{O}$ (Fig. 6 (c)). Earlier studies [65-66] showed that $\mathrm{H}_{2} \mathrm{O}$ at room temperature is similar to $\mathrm{D}_{2} \mathrm{O}$ if the temperature of the latter is raised by $\sim 5 \mathrm{~K}$ and the density increased by $\sim 0.5 \%$. However, the H/D substitution does not simply have an effect on the thermodynamical properties, there also exist geometrical differences between the structures of $\mathrm{H}_{2} \mathrm{O}$ and $\mathrm{D}_{2} \mathrm{O}$. Very recent results [64] have pinpointed that the intramolecular $\mathrm{O}-\mathrm{H}$ bond is longer by $\sim 3 \%$ than the corresponding $\mathrm{O}-\mathrm{D}$ bond length. In conjunction with the longer intramolecular $\mathrm{O}-\mathrm{H}$ bond in $\mathrm{H}_{2} \mathrm{O}$, the intermolecular $\mathrm{H}$ bond is shorter and more asymmetric than in $\mathrm{D}_{2} \mathrm{O}[54,64]$. Based on this $\mathrm{H}_{2} \mathrm{O}$ appears to have species where the strong donating $\mathrm{H}$ bonds are shorter as compared with $\mathrm{D}_{2} \mathrm{O}$. 

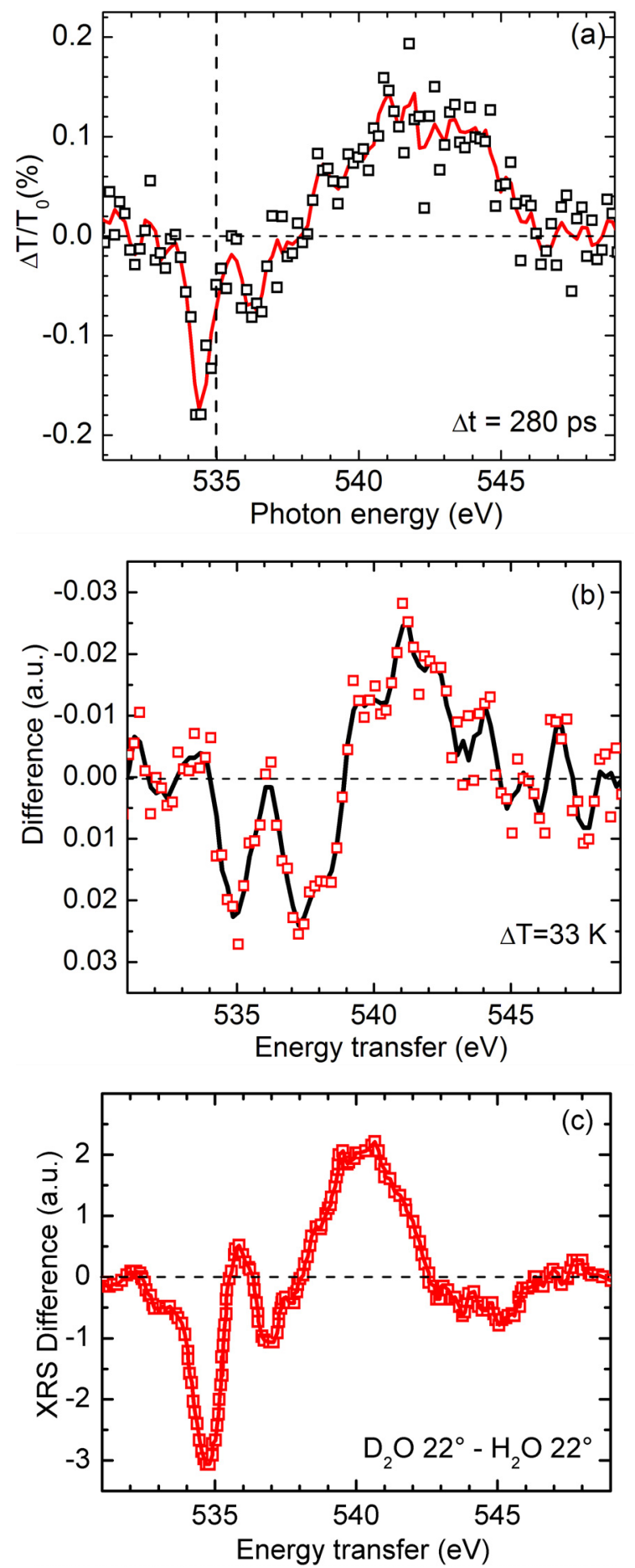

Fig. 6. (a) Transient changes of the x-ray transmission at a pump-probe delay time of 280 ps as a function of photon energy across the oxygen $K$ edge. $\Delta \mathrm{T}=\left(\mathrm{T}-\mathrm{T}_{0}\right)$, with $\mathrm{T}_{0}$ and $\mathrm{T}$ transmission before and after excitation. The spectrum is taken from [51]. (b) Changes of the x-ray Raman spectrum (energy resolution is $1 \mathrm{eV}$ ) for heating liquid water from 299 to $332 \mathrm{~K}$ (spectrum at $332 \mathrm{~K}$ minus spectrum at $299 \mathrm{~K})$. Measured raw data are shown as markers and smoothed data are shown as solid line. Data taken from [27]. Note that negative (positive) values are plotted to the top (bottom). (c) Changes of the x-ray Raman spectrum when comparing liquid $\mathrm{D}_{2} \mathrm{O}$ at 22 ${ }^{\circ} \mathrm{C}$ and liquid $\mathrm{H}_{2} \mathrm{O}$ at $22{ }^{\circ} \mathrm{C}\left(\mathrm{D}_{2} \mathrm{O}-\mathrm{H}_{2} \mathrm{O}\right)$. Data taken from [54].

The features in the time resolved transient spectral difference shown in Fig. 6 (a) could arise from a combination of the effects observed in steady-state heating and in isotopic substitution. The main edge decreases less than the pre and post edges when compared to steady-state heating but its change appears to be stronger than in the $\mathrm{H}_{2} \mathrm{O}-\mathrm{D}_{2} \mathrm{O}$ difference. This could indicate that ultrafast heating of liquid water entails two structurally distinct changes in the hydrogen bond network on a time scale of several hundreds of ps: 1) A change 
in the population of locally asymmetric and symmetric configurations and hence the weakening/breaking of hydrogen bonds and 2) An increase of the asymmetry of a certain fraction of molecules in asymmetric configurations by shortening of the strong donor hydrogen bond and elongation of the weak/broken donor hydrogen bond of the same molecule. However, for an average temperature increase of the excited volume of $16 \mathrm{~K}$, as derived from the absorbed infrared flux [51], a density change of $\sim 0.5 \%$ is expected. On a time scale of a few hundred ps after excitation, where we probe the transient x-ray absorption spectrum, the density has not changed yet. Therefore, when comparing the transient differences with steady-state heating and isotopic substitution one has to consider that in the later two cases the density change as due to temperature is included. In addition, because on ps time scale heating is taking place without change in volume the temperature change is associated with a pressure change. Only a few attempts have been made so far to investigate the effect of elevated pressures on the structure of liquid water [67] and on the near K-edge structure of oxygen [68]. However, these studies are not significant examples to allow for a quantitative estimation of the pressure induced changes in the O K-edge spectrum. Systematic investigations of the changes in the $\mathrm{O} \mathrm{K}$ edge spectrum on time scale out to several ns are planned in forthcoming experiments.

The long term overall temperature rise in our experiment is determined by the energy supplied via the infrared pump pulse. A quantitative analysis of the temperature jump can be made on the basis of the experimental parameters used for the transient data in Fig. 5: The measured pump pulse energy at the water film $(\sim 2.25 \mu \mathrm{J})$, the measured diameter of the pump beam on the sample $(210 \mu \mathrm{m})$, the measured water film thickness $(600 \mathrm{~nm})$, the tabulated absorption of the infrared radiation in water (OD $\sim 0.366$ ), and the tabulated heat capacity of liquid water $\left(\mathrm{Cp}=4.2 \mathrm{~J} / \mathrm{g}^{-1} \mathrm{~K}^{-1}\right)$. We also have to account for the different spot sizes of infrared pump and $\mathrm{x}$-ray probe beams.

Lateral heat diffusion leads to a spreading of excess energy and this has to be taken into account when estimating the size of the induced temperature jumps since it could lead to a temporal broadening of the temperature profile. Calculations show, however, that the lateral energy spreading in the water film occurs on a millisecond time scale [51], much longer than the time scale of our pump-probe study. Thus, the temperature profile as generated by the pump pulse is probed in the time-resolved experiments. It should be noted that the lateral energy spreading in $\mathrm{Si}_{3} \mathrm{~N}_{4}$ is substantially faster than in water (approximately by a factor of three), due to the higher heat diffusivity of $1.7 \times 10^{-2} \mathrm{~cm}^{2} / \mathrm{s}$ [69]. This fact indicates that most 
of the heat is, once transferred from the water film, dissipated through the $\mathrm{Si}_{3} \mathrm{~N}_{4}$ membranes before the next pump pulse impinges on the sample after $1 \mathrm{~ms}$.

From our experimental parameters we estimate a temperature increase of approximately $20 \mathrm{~K}$ averaged over the excited volume. However, the $\mathrm{x}$-ray probe-beam size is $115 \mu \mathrm{m}$ (horizontal) x $285 \mu \mathrm{m}$ (vertical) (Fig. 2 (c)) and this averages the size of the temperature jump over the excited volume and neighboring unpumped regions of the water film. Taking this into account, one again derives a temperature jump of 18 to $20 \mathrm{~K}$ averaged over the probed volume.

In our recently reported results [51] we showed that a measured transmission change of $0.15 \%$ in the pump-probe experiment corresponds to a temperature jump of $1 \mathrm{~K}$. Given this, one can independently estimate the temperature jump reported here: The $3.2 \%$ absorption change gives a temperature jump of $\sim 21 \mathrm{~K}$, in very good agreement with the temperature jump estimated from our experimental parameters.

\section{Summary and Conclusions}

We have presented a time-resolved x-ray absorption study of the structural dynamics in liquid water on a picosecond time scale. We report measured changes of x-ray absorption after an infrared induced temperature jump as large as $3.2 \%$. This corresponds to a temperature jump of approximately $20 \mathrm{~K}$. Associated structural changes are characterized by comparing a transient $\mathrm{x}$-ray absorption spectrum with spectral differences for steady-state heating and isotopic substitution in liquid water. Our results demonstrate the potential for combined infrared laser-synchrotron experiments with substantially shorter x-ray pulses such as generated with a femtosecond slicing scheme.

\section{Acknowledgments}

We thank Nils Huse for valuable support during the early stages of this experiment. We are grateful to the BESSY staff for continuous assistance and we thank in particular Karsten Holldack, Christian Stamm, Ulrich Schade, and Torsten Quast. We also gratefully acknowledge financial support from the Deutsche Forschungsgemeinschaft (SPP1134).

\section{References}

[1] D. Eisenberg, W. Kauzmann: The structure and properties of water, Oxford University Press, New York, 1969. 
[2] A. K. Soper, Chemical Physics 258, 121 (2000).

[3] T. Head-Gordon, G. Hura, Chem. Rev. 102, 2651 (2002).

[4] E. T. J. Nibbering, T. Elsaesser, Chem. Rev. 104, 1887 (2004).

[5] A. J. Lock, H. J. Bakker, J. Chem. Phys. 117, 1708 (2002).

[6] M. L. Cowan, B. D. Bruner, N. Huse, J. R. Dwyer, B. Chugh, E. T. J. Nibbering, T. Elsaesser, R. J. D. Miller, Nature 434, 199 (2005).

[7] S. Ashihara, N. Huse, A. Espagne, E. T. J. Nibbering, T. Elsaesser, J. Phys. Chem. A 111, 743 (2007).

[8] Z. Wang, Y. Pang, D. D. Dlott, J. Phys. Chem. A 111, 3196 (2007).

[9] Ph. Wernet, D. Nordlund, U. Bergmann, H. Ogasawara, M. Cavalleri, L. Å . Näslund, T. Hirsch, L. Ojamäe, P. Glatzel, M. Odelius, L. G. M. Pettersson, A. Nilsson, Science 304, 995 (2004).

[10] C. D. Cappa, J. D. Smith, K. R. Wilson, R. J: Saykally, J. Phys.: Condens. Matter 20, 205105 (2008).

[11] M. Odelius, M. Cavalleri, A. Nilsson, L. G. M. Pettersson, Phys. Rev. B 73, 024205 (2006).

[13] D. Prendergast, G. Galli, Phys. Rev. Lett. 96, 215502 (2006).

[14] D. Nordlund, H. Ogasawara, Ph. Wernet, M. Nyberg, M. Odelius, L. G. M. Pettersson, A. Nilsson, Chem. Phys. Lett. 395, 161 (2004).

[15] M. Cavalleri, H. Ogasawara, L. G. M. Pettersson, A. Nilsson, Chem. Phys. Lett. 364, 363 (2002). [16] H. Bluhm, D. F. Ogletree, C. S. Fadley, Z. Hussain, M. Salmeron, J. Phys.: Condens. Matter 14, L227 (2002).

[18] S. Myneni, Y. Luo, L.Å. Näslund, M. Cavalleri, L. Ojamäe, H. Ogasawara, A. Pelmenschikov, Ph. Wernet, P. Väterlein, C. Heske, Z. Hussain, L.G.M. Pettersson, A. Nilsson, J. Phys.: Condens. Matter 14, L213 (2002)

[19] B. Winter, E. F. Aziz, U. Hergenhahn, M. Faubel, I. V. Hertel, J. Chem. Phys. 126, 124504 (2007).

[20] J. D. Smith, C. D. Cappa, K. R. Wilson, B. M. Messer, R. C. Cohen, R. J. Saykally, Science 306, 851 (2004).

[21] O. Fuchs, M. Zharnikov, L. Weinhardt, M. Blum, M. Weigand, Y. Zubavichus, M. Bär, F. Maier, J.D. Denlinger, C. Heske, M. Grunze, and E. Umbach, Phys. Rev. Lett. 100, 027801 (2008)

[22] O. Fuchs, M. Zharnikov, L. Weinhardt, M. Blum, M. Weigand, Y. Zubavichus, M. Bär, F. Maier, J.D. Denlinger, C. Heske, M. Grunze, and E. Umbach Phys. Rev. Lett. 100, 249802 (2008).

[23] T. Tokushima, Y. Harada, O. Takahashi, Y. Senba, H. Ohashi, L.G.M. Pettersson, A. Nilsson, and S. Shin, Chem. Phys. Lett. 460, 387, (2008).

[24] J.-H. Guo, Y. Luo, A. Augustsson, J.-E. Rubensson, C. Såthe, H. Ågren, H. Siegbahn, and J. Nordgren, Phys. Rew. Lett. 89, 137402, (2002).

[25] T. Head-Gordon, S. W. Rick, Phys. Chem. Chem. Phys. 9, 83 (2007).

[26] M. V. Fernandez-Serra, E. Artacho, Phys. Rev. Lett. 96, 016404 (2006). 
[27] A. Nilsson, Ph. Wernet, D. Nordlund, U. Bergmann, M. Cavalleri, M. Odelius, H. Ogasawara, L.Å. Näslund, T. K. Hirsch, L. Ojamäe, P. Glatzel, L. G. M. Pettersson, Science 308, 793a (2005).

[28] J. D. Smith, C. D. Cappa, B. M. Messer, R. C. Cohen, and R. J. Saykally, Science 308 (2005) $793 b$.

[29] F. H. Stillinger, Science 209, 451 (1980).

[30] D. Marx, M. E. Tuckerman, J. Hutter, M. Parrinello, Nature 397, 601 (1999).

[31] L. X. Chen, W. J. H. Jager, G. Jennings, D. J. Gosztola, A. Munkholm, J. P. Hessler, Science 292, $262(2001)$.

[32] S. L. Johnson, P. A. Heimann, A. M. Lindenberg, H. O. Jeschke, M. E. Garcia, Z. Chang, R. W. Lee, J. J. Rehr, R. W. Falcone, Phys. Rev. Lett. 91, 157403 (2003).

[33] C. Bressler, M. Chergui, Chem. Rev. 104, 1781 (2004).

[34] A. Cavalleri, M. Rini, H. H. W. Chong, S. Fourmaux, T. E. Glover, P. A. Heimann, J. C. Kieffer, R. W. Schoenlein, Phys. Rev. Lett. 95, 067405 (2005).

[35] A. Lindenberg et al., Science 308, 392 (2005).

[36] W. Gawelda, M. Johnson, F. M. F. de Groot, R. Abela, C. Bressler, M. Chergui, J. Am. Chem. Soc. 128, 5001 (2006).

[37] C. Stamm, T. Kachel, N. Pontius, R. Mitzner, T. Quast, K. Holldack, S. Khan, C. Lupulsecu, E. F. Aziz, M. Wietstruk, H. A. Dürr, W. Eberhardt, Nature Materials 6, 740 (2007).

[38] S. L. Johnson, P. Beaud, C. J. Milne, F. S. Krasniqi, E. S. Zijlstra, M. E. Garcia, M. Kaiser, D. Grolimund, R. Abela, G. Ingold, Phys. Rev. Lett. 100, 155501 (2008).

[39] C. Gahl, A. Azima, M. Beye, M. Deppe, K. Döbrich, U. Hasslinger, F. Hennies, A. Melnikov, M. Nagasono, A. Pietzsch, M. Wolf, W. Wurth, A. Föhlisch, Nature Photonics 2, 165, (2008).

[40] C. Bressler, R. Abela, M. Chergui, Z. Kristallogr. 223, 307 (2008).

[41] L. Nugent-Glandorf, M. Scheer, D. A. Samuels, A. M. Mulhisen, E. R. Grant, X. Yang, V. M. Bierbaum, S. R. Leone, Phys. Rev. Lett. 87, 193002 (2001).

[42] M. Bauer, C. Lei, K. Read, R. Tobey, J. Gland, M. M. Murnane, H. C. Kapteyn, Phys. Rev. Lett. 87, 025501 (2001).

[43]T. Pfeifer, C. Spielmann, G. Gerber, Rep. Prog. Phys. 69, 443 (2006).

[44] C. v. Korff Schmising, M. Bargheer, M. Kiel, N. Zhavoronkov, M. Woerner, T. Elsaesser, I. Vrejoiu, D. Hesse, M. Alexe, Phys. Rev. Lett. 98, 257601 (2007).

[45] C. v. Korff Schmising, M. Bargheer, M. Woerner, T. Elsaesser, Z. Kristallogr. 223, 283 (2008).

[46] E. Gagnon, P. Ranitovic, X.-M. Tong, C. L. Cocke, M. M. Murnane, H. C. Kapteyn, A. S. Sandhu, Science 317, 1374 (2007).

[47] W. Fullagar, M. Harbst, S. Canton, J. Uhlig, M. Walczak, C.-G. Wahlström, V. Sundström, Rev. Sci. Instrum. 78, 115105 (2007).

[48] E. Collet, M. Buron-Le Cointe, M. Lorenc, H. Cailleau, Z. Kristallogr. 223, 272 (2008). 
[49] Taewoo Lee, Yan Jiang, Christoph G. Rose-Petruck, Frank Benesch, J. Chem. Phys. 122, 084506 (2005).

[50] V. C. Felicíssimo, F. F. Guimarães, F. Gel'mukhanov, A. Cesar, H. Ågren, J. Chem. Phys. 122, 094319 (2005).

[51] Ph. Wernet, G. Gavrila, K. Godehusen, C. Weniger, E. T. J. Nibbering, T. Elsaesser and W. Eberhardt, Appl. Phys. A 92, 511, (2008)

[52] J. D. Smith, C. D. Cappa, K. R. Wilson, B. M. Messer, R. C. Cohen, R. J. Saykally, Science 306, 851 (2004).

[53] L. A. Näslund, J. Lüning, Y. Ufuktepe, H. Ogasawara, Ph. Wernet, U. Bergmann, L. G. M. Pettersson, A. Nilsson, J. Phys. Chem. B 109, 13835 (2005).

[54] U. Bergmann, D. Nordlund, Ph. Wernet, M. Odelius, L. G. M. Pettersson, A. Nilsson, Phys. Rev. B 76, 024202 (2007).

[55] C. Laffon, S. Lacombe, F. Bournel, Ph. Parent, J. Chem. Phys. 125, 204714 (2006).

[56] B.L. Henke et. al. At. Data. Nucl. Data Tables 54,181 (1993). see also www-cxro.lbl.gov

[57] J. Stöhr, Nexafs Spectroscopy, Springer Verlag, Berlin Heidelberg, (1996).

[58] B. X. Yang and J. Kirz, Phys. Rev. B. 36, 1361 (1987)

[59] U. Bergmann, A. Di Cicco, Ph. Wernet, E. Principi, P. Glatzel, and A. Nilsson, J. Chem. Phys. 127, 174504 (2007).

[60] A. Nilsson, H. Ogasawara, M. Cavelleri, D. Nordlund, M. Nyberg, Ph. Wernet and L. G. M. Pettersson, J. Chem. Phys. 122, 154505 (2005).

[61] C. Laffon, S. Lacombe, F. Bournel, Ph. Parent, J. Chem. Phys. 125, 204714 (2006).

[62] Ph. Wernet, D. Testemale, J.-L. Hazemann, R. Argoud, P. Glatzel, L.G.M. Pettersson, A. Nilsson, U. Bergmann, J. Chem. Phys. 123, 154503 (2005).

[63] J. S. Tse, D. M. Shaw, D. D. Klug, S. Patchkovskii, G. Vankó, G. Monaco, and M. Krisch Phys. Rev. Lett. 100, 095502 (2008)

[64] A. K. Soper, C. J. Benmore, Phys. Rev. Lett. 101, 065502 (2008).

[65] J. H. Root, P. A. Egelstaff, and A. Hime, Chem. Phys. 109, 137 (1986).

[66] C. H. Cho, J. Urquidi, S. Singh and J. W. Robonson, J. Phys. Chem. B. 103, 1991 (1999).

[67] T. Radnai and H. Ohtaki Mol. Phys. 87, 103 (1996).

[68] Y. Q. Cai, H.-K. Mao, P. C. Chow, J. S. Tse, Y. Ma, S. Patchkovskii, J. F. Shu, V. Struzhkin, R. J. Hemley,H. Ishii, C. C. Chen, I. Jarrige, C. T. Chen, S. R. Shieh, E. P. Huang, and C. C. Kao, Phys. Rev. Lett. 94, 025502 (2005).

[69] X. Zhang, C. P. Grigoropoulos, Rev. Sci. Instrum. 66, 1115 (1995). 